\title{
Nachfrage aus dem „Westen“ trifft Arbeit aus dem „Süden“ PMSCs und der Einsatz von internationalen labour supply chains in der westlichen Kriegsführung
}

\author{
Hilde van Meegdenburg \\ Ludwig-Maximilians University, Munich
}

\section{Post-print version of:}

van Meegdenburg, Hilde. 2017. "Nachfrage aus dem »Westen« trifft Arbeit aus dem »Süden«: PMSCs und der Einsatz von internationalen labour supply chains in der westlichen Kriegsführung." Zeitschrift für Friedens- und Konfliktforschung 6(2): 289-308.

\section{https://doi.org/10.5771/2192-1741-2017-2-291}

\begin{abstract}
Private Militär- und Sicherheitsunternehmen (PMSCS) haben in den letzten Jahren viel Aufmerksamkeit erregt. Allerdings wurden sie bislang meist als Sicherheitsdienstleister angesehen und hinsichtlich ihrer Verantwortlichkeitsproblematik diskutiert. In diesem Essay beleuchte ich einen anderen, jedoch sehr wichtigen, Aspekt von PMSCs: Ihre Funktion als Arbeitsvermittler und die Bildung von internationalen Zuliefererketten von Arbeitskräften. Da die Nachfrage nach militärischen und unterstützenden Dienstleistungen größtenteils durch Arbeitskraft aus dem globalen Süden sichergestellt wird, konzentriere ich mich auf zwei Aspekte: (1) die Rekrutierungspraktiken von PMSCS und die Nutzung des globalen Reservoirs von Arbeitskräften; sowie (2) die Beschäftigung und Arbeitsbedingungen von Drittstaatenangehörigen in Afghanistan und dem Irak. Um die Beschäftigung von Drittstaatenangehörigen durch PMSCs in einen breiteren Kontext zu stellen, gehe ich kurz auf die Globalisierung von Produktion und Arbeit im Allgemeinen ein und folgere anschließend, dass diese Entwicklungen die Verteilung der sozialen, physischen und wirtschaftlichen Kosten und Nutzen von Kriegen verändert.
\end{abstract}

Schlagworte: Private Militär- und Sicherheitsunternehmen, labour supply chains, Kriegsbelegschaft, Beschäftigung von Drittstaatenangehörigen 


\section{Einleitung}

Private Militär- und Sicherheitsunternehmen (PMSCs) werden selten in Bezug auf ihre wohl wichtigste Aufgabe untersucht: Die Bereitstellung von Personal, das für die Ausführung der vom Militär ausgelagerten, meist sehr arbeitsintensiven Aufgaben, notwendig ist. Bereits heute leisten private Unternehmen, die ihre Mitarbeiterlnnen aus dem globalen Arbeitskräftereservoir beziehen, auf Militärbasen verschiedenste Dienste - von der Lebensmittelzubereitung bis hin zum Schutz der Stützpunkte. In der Vergangenheit wurden Militärbasen im Irak und in Afghanistan von Arbeitskräften aus Nepal, Peru und Uganda bewacht (Chisholm 2014: 356-357; Lucena 2013; Herbert 2016) und unter anderem von ArbeiterInnen aus Bangladesh, Indien, Pakistan und Sri Lanka bekocht und gereinigt (Barker 2009: 212-217; Cha 2004; Stillman 2011). Dies stellt nicht unbedingt eine negative Entwicklung dar. Jedoch handelt es sich um eine bedeutende Veränderung in der Organisation der Kriegsführung, die bislang weitgehend unbemerkt geblieben ist. Um es mit den Worten Amanda Chisholms auszudrücken: Trotz vereinzelter Aufmerksamkeit für die internationalen Arbeitskräfte, ist die Arbeit von Drittstaatenangehörigen (Third Country Nationals, TCNs) „largely excluded from academic and practitioner discussions" (Chisholm 2014: 351). ${ }^{1}$

Dieser Essay zielt darauf ab, TCNs und internationale labour supply chains als Forschungsthema zu erläutern und zu argumentieren, dass ihre Verbreitung die Verteilung der sozialen, physischen und wirtschaftlichen Kosten und Nutzen von Kriegen verändert. Zu diesem Zweck ist dieser Beitrag in zwei Abschnitte unterteilt. Zuerst untersuche ich die Beschäftigung von TCNs und die internationalen Zulieferketten von Arbeitskräften, über welche TCNs eingestellt werden. Anschließend gebe ich einen kurzen Überblick über die Globalisierung von Produktion und Arbeit im Allgemeinen, um die Beschäftigung von TCNs in einen breiteren Kontext zu stellen. Daran anschließend zeige ich anhand von Beispielen, wie die Internationalisierung der Kriegsbelegschaft die Verteilung der Kosten und Nutzen von Kriegen verändert. Dabei handelt es sich lediglich um erste empirische Erkenntnisse, die einen potentiellen Rahmen abstecken, in dem Veränderungen in der Kriegsführung untersucht und verstanden werden können.

1 Eine Ausnahme davon bilden feministische Betrachtungen der Hierarchien von Maskulinität und Ethnizität im privaten Sektor in Konfliktgebieten (Barker 2009; Higate 2012; Eichler 2014; Joachim/Schneiker 2015; Chisholm/Stachowitsch 2016). In dieser Literatur werden labour supply chains zwar erwähnt, der Fokus liegt jedoch auf der Durchsetzung von weißer militärischer Maskulinität im Vergleich zu, und durch die Unterordnung von, farbigen und feminisierten Arbeitskräften, welche explizit als „not soldiers” bezeichnet werden (Barker 2009: 228). 


\section{Nachfrage aus dem „Westen“ trifft Angebot aus dem „globalen Süden“}

Während PMSCs im Laufe der letzten Jahre viel Aufmerksamkeit zuteilwurde (so auch in dieser Zeitschrift, siehe Schneiker/Joachim 2012: 44-71), lag das Hauptaugenmerk meist auf Regulierungsund Rechenschaftsfragen (Leander 2013: 2-3; van Meegdenburg 2015: 329-332). Über die Rekrutierung ihres Personals und deren Arbeitsbedingungen ist dagegen weitaus weniger bekannt. Abgesehen von feministischen Ansätzen, welche die ethnische und geschlechtliche Unterordnung von TCNs in der sozialen Hierarchie von PMSCs darlegen (vgl. Joachim/Schneiker 2015; Chisholm/Stachowitsch 2016), hat Katherine M. McCoy (2010:676-678) dieses Thema bisher am umfangreichsten behandelt. Sie stellt fest, dass PMSCs eine "globale Reichweite" haben, die es ihnen erlaubt, eine „globale Belegschaft" aufzubauen (McCoy 2010: 676). Leider behandelt sie das Thema der Zulieferer- und Untervergabeketten, welche internationale Personalbeschaffung erst ermöglichen, nur grob und schlussfolgert, dass "this design works well for both PMCs and their clients" (McCoy 2010: 678). Was aber wissen wir über TCNs und ihre Arbeit in Konfliktgebieten?

\subsection{TCNs in Konfliktgebieten}

Im Jahr 2008 - dem ersten Jahr, für das diese Informationen verfügbar sind - gelangten allein durch die US-amerikanische Auftragsvergabe über 100.000 Menschen aus Drittstaaten in (PostKonfliktgebiete, in denen die USA militärisch aktiv waren (CENTCOM 2008: 1). ${ }^{2}$ Im Irak machten TCNs in diesem Jahr den zweitgrößten Teil des Personals aus: US-Amerikanische (Sub-)Unternehmen beschäftigten mehr als 72.000 Menschen aus Drittstaaten (CENTCOM 2008: 1). Zum Vergleich: Diese Zahl übersteigt die Anzahl der regulären Koalitionsstreitkräfte (minus den USA) bei Weitem. Laut dem „Iraq Index“ der Brookings Institution gab es im Januar 2008 im Irak 157.000 reguläre US-amerikanische Streitkräfte und 10.600 Koalitionsstreitkräfte. Entsprechende Zahlen variieren je nach Jahr und Kriegsschauplatz (siehe Tabellen 1 und 2). Dabei ist es unwahrscheinlich, dass sie das gesamte Ausmaß von TCN-Arbeit erfassen. ${ }^{3}$

2 Das US-amerikanische Program Support begann seine Arbeit Ende 2006. Die erste verfügbare Publikation des Quarterly Contractor Census Reports, welche die US-amerikanische Beschäftigung von SubunternehmerInnen inklusive TCNs untersucht, bezieht sich auf das dritte Quartal des Jahres 2008 (CENTCOM 2008).

3 Die Zahlen sind zum einen nicht eindeutig, da das Ausmaß und die Undurchsichtigkeit der Lieferketten es für die US-amerikanischen Behörden schwermachen, die genaue Zahl von Angestellten in Großverträgen zu bestimmen (Schwartz/Swain 2011). Zum anderen beinhalten diese Zahlen nur die Auslagerungen seitens der USA und nicht die von anderen Staaten und internationalen Organisationen. Wenngleich in geringerem Maße als die USA, lagern auch Staaten wie Dänemark, Deutschland, Frankreich, Kanada, Norwegen, Schweden und Großbritannien Teile ihrer Militärfunktionen aus (Spearin 2005; Petersohn 2010; Mandrup 2013; Krahmann 2013; Østensen 2013; Olsson 2013; Kruck 2014). 
Tabelle 1: Streitkräfte, irakische Beschäftigte, und Drittstaatenangehörige im Irak

\begin{tabular}{lllll}
\hline & $\begin{array}{l}\text { US-amerikanische } \\
\text { Streitkräfte }\end{array}$ & Koalitionsstreitkräfte & $\begin{array}{l}\text { Irakische } \\
\text { Staatsangehörige }\end{array}$ & $\begin{array}{l}\text { TCNs der US- } \\
\text { amerikanischen } \\
\text { Streitkräfte }\end{array}$ \\
\hline 2008 & 157.000 & 10.604 & 63.292 & 72.109 \\
2009 & 142.000 & 7.000 & 30.007 & 53.780 \\
2010 & 110.000 & - & 10.668 & 42.457 \\
2011 & 47.000 & - & 7.370 & 29.213 \\
2012 & - & - & 2.065 & 4.621 \\
\hline
\end{tabular}

Quelle: eigene Darstellung der Autorin basierende auf: Brookings Institute „Iraq Index“ (2012a: 13); CENTCOM (2008: 1); CENTCOM (2009: 1); CENTCOM (2010: 1); CENTCOM (2011: 1); CENTCOM (2012: 1)

Tabelle 2: Streitkräfte, afghanische Beschäftigte, und Drittstaatenangehörige in Afghanistan

\begin{tabular}{lllll}
\hline & $\begin{array}{l}\text { US-amerikanische } \\
\text { Streitkräfte }\end{array}$ & Koalitionsstreitkräfte & $\begin{array}{l}\text { Afghanische } \\
\text { Staatsangehörige }\end{array}$ & $\begin{array}{l}\text { TCNs der US- } \\
\text { amerikanischen } \\
\text { Streitkräfte }\end{array}$ \\
\hline 2008 & 27.500 & 28.250 & 58.466 & 4.381 \\
2009 & 34.400 & 31.880 & 78.430 & 16.349 \\
2010 & 71.700 & 38710 & 34.222 & 15.503 \\
2011 & 97.000 & 41.893 & 50.687 & 27.912 \\
2012 & 89.000 & 40.386 & 38.270 & 39.480 \\
\hline
\end{tabular}

Quelle: eigene Darstellung der Autorin basierende auf: Brookings Institute „Afghanistan Index“ (2012b: 4); CENTCOM (2008: 1); CENTCOM (2009: 1); CENTCOM (2010: 1); CENTCOM (2011: 1); CENTCOM (2012: 1)

Leider gibt das US-amerikanische Central Command keine Auskunft über die Herkunftsländer der Beschäftigten. Die folgende Liste gibt jedoch zumindest einen groben Überblick: Die Arbeitsgruppe der Vereinten Nationen für die Verwendung von SöldnerInnen (nachfolgenden „UN-Arbeitsgruppe“) zählte unter den TCNs, die im Irak als Sicherheitsdienstleister tätig waren mindestens 14 verschiedene 
Nationalitäten. ${ }^{4}$ Demnach gab es neben „expatriates" aus Australien, Kanada und Großbritannien unter anderem Beschäftigte aus Bulgarien, Kolumbien, Chile, El Salvador, Fidschi, Honduras, Nepal, Peru, den Philippinen, Rumänien, Russland und der Ukraine. Außerdem gibt die UN-Arbeitsgruppe an, „zuverlässige Informationen“ zu besitzen, wonach BürgerInnen aus Nepal, Fidschi, Singapore, den Philippinen und Nigeria militärische Funktionen in der Unterstützung der Streitkräfte der Vereinigten Staaten in Afghanistan innehatten (UNHRC 2008a: 12). Isabella Barker (2009: 214) sowie verschiedene Zeitungsartikel (vgl. Cha 2004; Stillman 2011) berichten von vielen Beschäftigten aus Bangladesch, Indien, Fidschi, Pakistan und Sri Lanka im Verpflegungs- und Reinigungssektor sowie von Wachposten aus Uganda (Herbert 2016; Lawrence 2016). Laut dem letzten CENTCOM-Report gibt es auch 2017 noch 10.279 TCNs, welche für US-Amerikanische Streitkräfte und (Sub-)Unternehmen in Afghanistan und dem Irak arbeiten (CENTCOM 2017: 1).

Ohne Übertreibung kann man daher sagen, dass zehntausende Menschen aus dem globalen Süden in Konfliktgebiete gebracht wurden, um dort die westliche Kriegsführung zu unterstützen. Obwohl PMSCs selbst oft mit ehemaligen britischen und US-amerikanischen Eliteeinheiten als Teil ihre Kriegsbelegschaft werben (Kruck/Spencer 2013: 332-334; Joachim/Schneiker 2015: 115), besteht ein großer Teil der Angestellten also aus TCNs. Ebenso arbeitet die überwiegende Mehrheit nicht im Sicherheitsbereich - obwohl den Unternehmen in diesem Bereich bislang am meisten Aufmerksamkeit zuteilwurde (van Meegdenburg 2015: 337-339; vgl. Barker (2009) als Ausnahme). Laut Statistiken des US-amerikanischen Zentralkommandos waren im Jahr 2008 in Afghanistan nur 5,6\% und im Irak nur 6,4\% der gesamten privaten Belegschaft als Sicherheitsdienstleister tätig (CENTCOM 2008: 2-3). Auch hinsichtlich der TCNs war im Schnitt nur eine Minderheit des privaten Personals mit Sicherheitsfunktionen betraut (siehe Tabelle 3) - wenngleich ihr Anteil an Sicherheitsdienstleistern im Irak signifikant höher ist. ${ }^{5}$

4 Der vollständige Name der Arbeitsgruppe lautet: „UN Human Rights Council Working Group on the use of mercenaries as a means of violating human rights and impeding the exercise of the right of people to self-determination" (UNHRC 2008a).

$5 \quad$ Diese Zahlen basieren auf den Anteilen der Beschäftigten, welche von dem US-amerikanischen Department of Defence als Private Security Contractors (PSC) aufgeführt werden, im Vergleich zur Gesamtzahl der Beschäftigten an diesen Schauplätzen pro Jahr. Der Anteil der Sicherheitsdienstleister unter den TCNs in Afghanistan ist geringer, da hier viele lokale Angestellte beschäftigt wurden, um diese Funktionen auszuführen. Im Jahr 2012 waren ca. 38.270 Afghanlnnen beschäftigt, wovon 15.413 (40,2\%) als Sicherheitsdienstleister aufgeführt sind (CENTCOM 2012: 1-2). 
Tabelle 3: TCN-Beschäftigung in Afghanistan und im Irak - Anteil der Sicherheitsdienstleister

\begin{tabular}{lllllll}
\hline & $\mathbf{2 0 0 8}$ & $\mathbf{2 0 0 9}$ & $\mathbf{2 0 1 0}$ & $\mathbf{2 0 1 1}$ & $\mathbf{2 0 1 2}$ \\
\hline Afghanistan & TCNs & 4.381 & 16.349 & 15.503 & 27.912 & 39.480 \\
& davon Sicherheitskräfte & 32 & 1.017 & 858 & 948 & 1.437 \\
& prozentual & $0,7 \%$ & $6,2 \%$ & $5,5 \%$ & $3,4 \%$ & $3,6 \%$ \\
Irak & & & & & 4.621 \\
& TCNs & 72.109 & 53.780 & 42.457 & 29.213 & 1.823 \\
& davon Sicherheitskräfte & 8.188 & 9.212 & 9.713 & 8.293 & $39,5 \%$ \\
\hline
\end{tabular}

Quelle: eigene Darstellung der Autorin basierende auf: CENTCOM (2008: 1-2); CENTCOM (2009: 1-2); CENTCOM (2010: 1-2); CENTCOM (2011: 1-2); CENTCOM (2012: 1-2)

Wenn man zusätzlich bedenkt, dass das Auslagern von anderen (vor allem europäischen) Staaten als "weniger umfangreich" beschrieben wird (Krahmann 2010: 39) und sich größtenteils auf Unterstützungsfunktionen beschränkt (vgl. die Fallstudien in Leander 2013), kann geschlussfolgert werden, dass die überwiegende Mehrheit des privaten Personals nicht in militärischen Funktionen aktiv ist, sondern sich mit der Versorgung der Basis, Übersetzungen, Transport und Wartungsfunktionen beschäftigt. Die bisherige Fokussierung auf Sicherheitsdienstleister sowie Regulierungs- und Rechenschaftsfragen in der Literatur könnte daher eine wichtige Entwicklung in Bezug auf die Beschäftigung von PMSCs verschleiert haben: Nämlich, dass die Nachfrage nach militärischen und unterstützenden Dienstleistungen aus dem Westen größtenteils durch Arbeitskraft aus dem globalen Süden befriedigt wird.

\subsection{Subunternehmen und die Rekrutierung des privaten Kriegspersonals}

Die Ketten von Subunternehmen werden sowohl in der Forschung als auch in der Politik primär in Verbindung mit der damit einhergehenden Verantwortlichkeitsproblematik thematisiert (siehe $u$. a. Schwartz/Swain 2011; McCoy 2010: 676-678; Perry 2012: 43; Berndtsson 2014: 556-557). Ihre Funktion als labour supply chains wird hingegen selten diskutiert. Zu einem großen Teil sind Subunternehmen aber genau das: Unter Zuhilfenahme des globalen Reservoirs von Arbeitslosigkeit und Unterbeschäftigung versorgen sie Konfliktgebiete mit meist schlecht bezahlten ArbeiterInnen. McCoy argumentiert: „The PMC industry has been able to rapidly expand in recent years because of this ability to quickly draw on and organize large work forces with a variety of skill sets, experiences, and wage expectations from around the globe" (2010: 678). Zugleich ist es aus arbeitsrechtlicher Perspektive wahrscheinlich, dass diese Ketten, gerade weil sie Arbeitsbeziehungen und Verantwortlichkeiten verschleiern, nachteilige Auswirkungen auf die Arbeitsbedingungen und 
ArbeitnehmerInnenrechte in diesem Wirtschaftszweig haben (UNHRC 2007: 2; UNHRC 2008a: 7-8). Nachfolgenden werden labour supply chains an Hand von einigen Beispielen genauer veranschaulicht. Im nächsten Abschnitt wird auch ihr Einfluss auf ArbeiterInnen und deren Rechte diskutiert und die Umverteilung der Kosten und Nutzen der Kriegsführung hervorgehoben.

Die UN-Arbeitsgruppe stellte fest, dass chilenische, honduranische und peruanische Staatsangehörige durch lokal tätige Personalvermittlungen, Tochtergesellschaften und Agenturen als Sicherheitsbedienstete im Irak eingestellt wurden. Damit bildeten diese VermittlerInnen ein Netz von Subunternehmen, das sich über mehrere territoriale und regulative Regime erstreckte. Die vielleicht direkteste Ausgestaltung eines solchen Netz von Subunternehmen zeigt sich am Beispiel von Triple Canopy und der Einstellung von RekrutInnen aus Honduras: Triple Canopy, ein US-amerikanischer Hauptauftragnehmer mit Aufgabe der Bewachung der "green zone" in Bagdad, vergab einen Unterauftrag zur Organisation der Arbeitskraft an eine US-amerikanische Personalvermittlung namens Your Solutions Inc. Dieses Subunternehmen aus Illionis gründete eine Tochtergesellschaft in Honduras, Your Solutions Honduras, um genügend Arbeitskräfte zu finden und auszubilden (UNHRC 2007: 7-12). Triple Canopy zahlte den honduranischen Sicherheitsbediensteten $990 \$$ pro Monat.

Das Netz von Subunternehmen, durch welches die chilenischen Sicherheitsbediensteten rekrutiert wurden, war verzweigter und undurchsichtiger. Die UN-Arbeitsgruppe stellte fest, dass die im Irak für Blackwater und Triple Canopy arbeitetenden ChilenInnen durch Personalagenturen aus Uruguay und Panama eingestellt wurden. Diese wurden extra gegründet, um Probleme mit den chilenischen Behörden zu vermeiden (UNHRC 2008b: 9). Eine zentrale Rolle in dieser Kette nehmen vier Unternehmen eines einzelnen Eigentümers ein: José Miguel Pizarro. Pizarro organisierte einen Teil der Arbeitskräfte für Blackwater und Triple Canopy durch die Unterfirmen Red Táctica Inc. mit Sitz in den USA; Grupo Táctico Chile mit Sitz in Santiago de Chile; Neskowin mit Sitz in Urugay; sowie Global Guards mit Sitz in Panama (UNHRC 2008b). Neben anderen Unregelmäßigkeiten stellte die UN-Arbeitsgruppe fest, dass die Triple Canopy-Verträge angeblich in Amman, Jordanien, die Blackwater-Verträge dagegen in North Carolina, USA, unterzeichnet wurden. Dies, zusammen mit einer Zahl an weiteren in den Verträgen enthaltenen Klauseln, führte die UN-Arbeitsgruppe zu folgendem Schluss:

„By signing these contracts, Chileans were not only renouncing some of their most fundamental rights - such as the right to be subject to their country's laws, given that its courts did not have jurisdiction - but were also, in effect, incapacitating themselves in the event they had to file a claim against the company" (UNHRC 2008b: 10). 
Während Blackwater seinem Personal monatlich etwa 3.000\$ bezahlte, erhielten ChilenInnen später von Triple Canopy $990 \$$ pro Monat. ${ }^{6}$

Die labour supply chains, durch die Arbeitskräfte aus asiatischen und pazifischen Ländern ihren Weg in Konfliktregionen fanden, sind komplexer und beinhalten größere Risiken für die ArbeitnehmerInnen. Besorgniserregend ist insbesondere, dass hier oft lokale „AgentInnen“ und „PersonalvermittlerInnen“ Rekrutierungsgebühren erheben und RekrutInnen durch falsche Job- und Bezahlungsversprechen in die Irre führen. Wie sehen diese labour supply chains in der Praxis aus?

Ariana Cha (2004) deckte eine Kette von Subunternehmen auf, welche indische ArbeitnehmerInnen für Kellog, Brown \& Root (KBR) rekrutierte. ${ }^{7}$ Ausgehend von einer lokalen indischen Personalvermittlung, beinhaltete diese „five levels of subcontractors and employment agents

“ (Cha 2004). Cha erzählt die Geschichte des 29-jährigen Dharmapalan Ajayakumar aus Kerala, Indien. Ajayakumar zahlte einem Agenten, der ihm ein Stellenangebot für zwei Jahre Catering in Kuwait gemacht hatte, im Vorhinein 1.800\$ für die Rekrutierung. Wie Cha erklärt, lieh er das Geld von lokalen „Kredithaien“. Zusammen mit anderen indischen Servicekräften (Köche und Köchinnen, LKWFahrerInnen und BauarbeiterInnen) wurde er ursprünglich durch eine Personalvermittlung namens Subhash Vijay eingestellt, welche inn wiederum engagiert hatte "to work for Gulf Catering Co. of Riyadh, Saudi Arabia, which was subcontracted to Alargan Group of Kuwait City, which was subcontracted to the Event Source of Salt Lake City, which in turn was subcontracted to KBR of Housto" (Cha 2004) . Erst als die indischen Servicekräfte in Kuwait angekommen waren, wurden sie informiert, dass sie nicht dort, sondern im Irak arbeiten sollten. Letzten Endes wurden sie in einer amerikanischen Basis im Nordirak eingesetzt: Qayyarah Airfield West. Ajayakumar und seine KollegInnen bekamen ihren Angaben zufolge „less than \$7 a day", was bei einer Sechstagewoche ungefähr 200\$ im Monat entspricht (Cha 2004).

Ähnlich wie im Fall Ajayakumars berichtet Stillman (2011) von Fidschianerinnen, die im Vorhinein 500\$ bezahlten, angeblich um als Personal für einen Schönheitssalon in Dubai angestellt zu werden. Nachdem sie von der Meridian Services Agency rekrutiert worden waren, stellte sich allerdings heraus, dass sie an eine türkische Firma namens Kulak Construction weitervermittelt worden waren, die sie zu dem US-amerikanischen Basis Camp Anaconda im Norden Bagdads brachte. Im Camp Anaconda angekommen erfuhren die Frauen, dass sie nicht wie versprochen $1.500 \$$ monatlich verdienen würden. Stattdessen „the women were told that they would make only seven hundred dollars a month, a sum that was later reduced, under another subcontractor, to three hundred and fifty" (Stillman

6 Leider bietet die UN-Arbeitsgruppe keine Erklärung für diese unterschiedliche Vergütung. In einem Artikel für The North American Congress on Latin America (NACLA, eine gemeinnützige Organisation) argumentiert Eric Stoner aber, die Diskrepanz könne darauf zurückgeführt werden, dass Blackwater als erstes in den Markt eingestiegen sei. Nachdem Pizarro ebenfalls begann, für Triple Canopy zu rekrutieren und lediglich $\$ 990$ bot, wurde dies zum „,going rate for recruits throughout the region " (Stoner 2014). 
2011). Anfänglich traten die Frauen in Streik. „It was ridiculous, really, slave labor, absolutely ridiculous out here in a war zone", zitiert Stillman eine der Frauen (2011). Laut Stillman akzeptierten die Frauen nach zwei Wochen Streik einen Vertrag, der ihnen $800 \$$ monatlich versprach.

Da verschiedene Subunternehmen eine Vielzahl an Versorgungsketten etabliert haben, gibt es wahrscheinlich viele weitere Fälle dieser Art. Wie Cha hervorhebt: „there are no restrictions on the number and 'tiers' of subcontractors that can be used" (Cha 2004). Daraus resultieren wichtige Vorteile auf Seiten der Firmen: Derart komplexe Arbeitsverträge sind für ArbeiterInnen schwer durchschaubar. So argumentiert José L. Gómez del Prado (ehemaliger Vorsitzender der UN Working Group on Mercenaries), dass entsprechende Zuliefererketten viele rechtliche Unklarheiten schaffen und MitarbeiterInnen in eine Position versetzen, in der Rechtsstreitigkeiten unglaubliche Herausforderungen und Kosten mit sich brächten (UNHRC 2008b). Darüber hinaus profitieren die Firmen davon, dass Lohn und Arbeit nur den Anforderungen des Landes entsprechen müssen, in dem die „letzte“ Agentur der labour supply chain ihren Sitz hat (McCoy 2010: 676-677).

Es gibt einige Anzeichen dafür, dass die Vorgehensweise der UnterauftragnehmerInnen vergleichbar ist, wenn sie von NATO-Mitgliedern oder anderen Staaten beauftragt werden. Zum Beispiel erwähnte Roger Maclsaac, ehemaliger Projektmanager der ATCO Structures and Logistics auf dem Flughafen Kandahar, dass ATCO Frontec für die NATO neben „ehemaligen Militärangehörigen“ aus NATO-Staaten auch Arbeitskräfte aus „wahrscheinlich zehn anderen Ländern" beschäftigte, wobei viele „aus Sri Lanka, Südafrika, den Philippinen, Rumänien und Kroatien“ kamen (Maclsaac, April 2014, Ottawa). ${ }^{8}$ Weiter findet sich beispielsweise im kanadischen Chief Review Services Bericht, dass 200 NepalesInnen unter dem Canadian Contractor Augmentation Program für den kanadischen Hauptauftraggeber SNCLavalin gearbeitet haben (Chief Review Services 2006: 8). Und auch im dänischen Parlament wurden Fragen diskutiert, die deutlich machen, dass Supreme Foodservices unter dänischen Verträgen unterbezahlte TCNs beschäftigte (Folketing 2007). ${ }^{9}$

\section{Die Globalisierung von Produktion und Arbeit}

Die Frage, warum Staaten, die NATO und die Vereinten Nationen Outsourcing betreiben, wurde in der Literatur bereits diskutiert (vgl. u. a. Spearin 2003; Krahmann 2010; Petersohn 2010; Berndtsson 2014; Kruck 2014). Hier bette ich die Beschäftigung von TCNs in einem breiteren Kontext ein und erörtere

8 Ich interviewte Herrn Maclsaac im April 2014 in Ottowa bezüglich meiner Forschung zum Outsourcing im kanadischen Militär. 
die Organisation von Netzen von Subunternehmen und labour supply chains im Allgemeinen. Ich gehe der Frage nach, welchen Einfluss diese auf ArbeiterInnen und deren Rechte haben. Die folgende Analyse soll nicht als kausales Argument betrachtet werden. Vielmehr soll sie einen potentiellen Rahmen bieten, in dem Veränderungen in der Kriegsführung untersucht und verstanden werden können.

\subsection{Die Globalisierung von Produktion und Arbeit im Allgemeinen}

Globalisierung wird häufig als eine treibende Kraft für die internationale Reorganisation von Produktion und Arbeit dargestellt (vlg. Henderson et al. 2002; 438; Kalleberg 2009: 5; Mosley 2011: 3, 11-14; Coe/Hess 2013: 4). Die Globalisierung der Produktion, die direkt - durch Auslandsdirektinvestitionen (ADI) - oder indirekt - durch Outsourcing, Untervergabe und die Etablierung globaler Produktionsnetzwerke (GPNs) - zustande kommt, kann weder als durchweg positiv noch negativ bewertet werden. Während ADI, welche sich ohne die Hilfe von ZwischenhändlerInnen und lokalen Vertragsfirmen organisieren, als Anreiz für Unternehmen gesehen werden, in die Bildung, Gesundheit und den allgemeinen sozialen Wohlstand ihrer ArbeiterInnen zu investieren, haben Subunternehmen und Arbeitsvermittlungen häufig dem entgegengesetzte Absichten und Effekte (Mosley/Uno 2007: 925-928; Mosley 2011: 12-13). Besonders Untervergabeverfahren, mit ihrer Tendenz zur Neuvergabe von Aufträgen und der Suche nach Flexibilität und kostengünstigster Produktion, wird nachgesagt, den Wettbewerb zwischen Staaten und Regionen zu verstärken (Mosley/Singer 2014: 285-286). Dies gilt im Besonderen für arbeitsintensive Industriezweige, die primär geringqualifizierte Arbeitskräfte benötigen (Mosley 2011: 12). Das häufig erwähnte "race to the regulatory bottom" wird deshalb nicht im Kontext der Globalisierung per se, sondern speziell im Zusammenhang mit der Untervergabe von Aufträgen und der Arbeitsvermittlungspraxis diskutiert (Mosley 2011: 8-10).

Trotzdem müssen Untervergabeverfahren und internationale Arbeitskräfteanwerbung nicht zwangsläufig einen negativen Einfluss haben. Die Anwerbung von Arbeitskräften aus Niedriglohnregionen und die Nutzung von WanderarbeiterInnen kann zur Verbesserung der Wohlfahrt jener führen, die über keine anderen Ressourcen als ihre eigene Arbeitskraft verfügen. Ob dies tatsächlich geschieht, hängt von der Beschäftigungsart und den Umständen ab, unter denen das Arbeitsverhältnis begonnen wird (Barrientos 2013: 1058). Ein positiver Effekt von Arbeitsmigration und internationaler Arbeitsvermittlung ist, dass dadurch die direkte Umverteilung von Wohlstand, Wissen und Fähigkeiten ermöglicht wird. Devesh Kapur (2010) betrachtet die Bedeutung von Migration und Geldsendungen als einen bisher vernachlässigten Faktor der Globalisierung. Er argumentiert, dass der "flow of labour" aus einigen Staaten hin zu anderen immense Vorteile für den entsendenden Staat haben kann, da es Familien und Gemeinschaften erlaubt, in ihre soziale und ökonomische Existenz zu investieren (Kapur 2010: 5-6, vgl. Castles 2010; Kelly 2013; Mosley/Singer 2014). 
Allgemein ist festzustellen, dass die Globalisierung die Verteilung sozialer, physischer und ökonomischer Kosten und Nutzen der Produktion zwischen Staaten und lokalen Gesellschaften verändert hat. Es scheint so, als könne ein ähnliches Fazit auch in Bezug auf die internationalen labour supply chains, welche westliche Kriegsführung unterstützen, gezogen werden: Sie verändern die Verteilung der sozialen, physischen und wirtschaftlichen Kosten und Nutzen der Kriegsführung.

\subsection{Die Globalisierung der Kriegsbelegschaft und die Verteilung der Kosten und Nutzen}

Zweifellos profitieren einige Angestellte von PMSCs von ihrer Arbeit in Afghanistan oder dem Irak ungeachtet der Risiken. So berichtete der Peruaner Norman Alfonso Solano einem Reporter: „hier [in Lima] verdiene ich 200\$ pro Monat, wenn es Arbeit gibt“ (Páez 2007, eigene Übersetzung). Da es in Peru immer schwieriger wurde Arbeit zu finden, verpflichtete er sich entgegen der Ängste seiner Familie schließlich für ein Arbeitsverhältnis im Irak. „Ich hatte sehr großes Glück, nicht jeden Tag bekommt man die Möglichkeit, so viel Geld zu verdienen“ (Páez 2007, eigene Übersetzung). Nach seiner ersten Verpflichtung war die Familie in der Lage ein neues Haus zu bauen und das Schulgeld für die Kinder zu bezahlen (Páez 2007). In Anlehnung daran argumentiert auch Rolando Soto, ein ehemaliger Personalvermittler von Triple Canopy in Peru: Diejenigen AuftragnehmerInnen, die durch ihren im Ausland erworbenen Lohn in ihrem Heimatland ein Haus bauen oder ihre Kinder auf bessere Schulen schicken konnten, waren dankbar dafür (Lucena 2013). „Ihre Lebensqualität hat sich merklich verbessert" (Soto in Lucena 2013, eigene Übersetzung).

Erwerbstätigkeit im Ausland und Lohnsendungen können einen großen Einfluss haben, insbesondere auf Gesellschaften und Regionen mit hohen Arbeitslosigkeits- und Armutsraten. Eine Aussage des fidschianischen Arbeitsminister Kenneth Zinck verdeutlicht, wie attraktiv entsprechende Erwerbsmöglichkeiten und Lohnsendungen durch PMSCs auch für die Entsendestaaten sind: „the Government knows that more men are leaving for Kuwait and Iraq and it is a good thing because it is providing employment for the unemployed. This is one solution to the increasing unemployment rate in the country today" (Maclellan/Mares, 2006: 143). Ein anderes Beispiel ist Uganda, wo "returning guards use earnings to buy land, build homes, and start small businesses" (Herbert 2016) und wo, laut dem Arbeitsminister Mwesigwa Rukutana, „the Iraq opportunity brings in about $\$ 90$ million dollars, whereas our chief export, which is coffee, brings in around $\$ 60$ or $\$ 70$ million a year" (Delany 2009). Für einige bedeutet die Beschäftigung im Irak oder Afghanistan jedoch nur eine vorübergehende oder gar keine Verbesserung. Solano zum Beispiel verpflichtete sich erneut, da sein Geld bald ausgegeben war und sich die Lage in Lima nicht verbessert hatte. Und das, obwohl er bei seinem ersten Aufenthalt im Irak jeden Tag Angst um sein Leben hatte (Páez 2007).

Er berichtete zudem, dass seine KollegInnen bei Angriffen getötet oder lebensbedrohlich verletzt wurden (Páez 2007). Und während reguläre Streitkräfte und ihre Familien oft durch spezielle Militär- 
Wohlfahrtssysteme gegen Todesfälle und Verletzungen versichert sind (Cowen 2006: 177), haben ausländische AuftragnehmerInnen große Schwierigkeiten ihre Rechte, etwa auf Schmerzensgeld, durchzusetzen (UNHCR 2008b: 10; Miller 2009; 2010). Thematisiert wird diese Problematik unter anderem in dem Dokumentarfilm America's 33\$ dollar mercenaries von Fernando Lucena (2013). Der Film berichtet von Peruanern, welche für Triple Canopy und die US-Amerikanischen Streitkräfte arbeiteten und mit verschiedenen Verletzungen aus dem Irak zurückkehrten. In einem Interview erklärt José Gómez del Prado: „when they are injured, in order to get the compensation, is hell because you have to claim to the United States" (Goméz del Prado, in Lucena 2013). Da sich viele Versicherungen weigern, geforderte Entschädigungen auszuzahlen, müssen sich viele AuftragnehmerInnen nach ihrer Rückkehr mit kostenintensiven und organisatorisch schwierigen Rechtsstreitigkeiten auseinandersetzten (Goméz del Prado, in Lucena 2013, vgl. Lawrence 2016).

Für diejenigen Beschäftigten, die verletzt (oder sogar tot) zurückkehren, birgt die Arbeit im Kriegsgebiet einen hohen körperlichen und damit häufig auch einen hohen sozialen und ökonomischen Preis. Durch Verletzung werden zukünftige Beschäftigungen häufig schwierig oder gar unmöglich (vgl. Lucena 2013; Lawrence 2016). Weigert sich dann auch noch die Versicherung für den entstandenen Schaden einzustehen, muss in manchen Fällen die Familie oder Gemeinschaft für notwendige Operationen aufkommen. ${ }^{10}$

Zu den beschriebenen Gefahren, die sich aus der Beschäftigung in Konfliktregionen ergeben, kommen weitere Problematiken, die aus der Untervergabe von Aufträgen resultieren. Wie aus der Globalisierung von Produktion und Arbeit in anderen Industrien bekannt ist, tendieren insbesondere Zulieferketten und Arbeitsvermittlungen dazu, durch die Neuvergabe von Aufträgen und die fortwährende Suche nach Flexibilität und kostengünstiger Produktion, Investition in Bildung, Gesundheit und den allgemeinen sozialen Wohlstand ihrer Arbeitskräfte zu vernachlässigen. Dies trifft auch für die Militärindustrie zu.

Selbst für die relativ kurze Untervergabekette, in der Honduraner mittels Your Solutions für Triple Canopy arbeiteten, bekam die UN-Arbeitsgruppe Informationen Fall von Verletzungen über ihren Schweregrad) möglich. „Although they outnumbered Americans by about 2-to-1, the thirdcountry nationals filed just 14 percent of the total claims", was darauf hindeutet, "that many wounded foreigners never apply for benefits" (Miller 2009; vgl. auch Lawrence 2016). Wie Christian T. Miller betont, entziehen sich außerdem viele ArbeitgeberInnen ihrer Verantwortung, Sterbe- und Verletzungsfälle zu dokumentieren. Bekannt ist nur, dass zwischen 2001 und 201628.784 Schadensfälle von TCNs gemeldet wurden, wovon 476 Todesfälle waren. Es ist jedoch nicht bekannt, inwieweit diese Zahlen die tatsächlichen Schadens- und Todesfälle widerspiegelt. Für die Defence Base Act Summary Reports siehe: Webseite United States Department of Labour. 
concerning irregularities in compliance with the contracts of these employees, who report harsh working conditions, partial payment or nonpayment of wages, arbitrary action by superiors including isolation, lack of basic necessities such as food and lodging, as well as a lack of medical treatment and poor sanitation (UNHRC 2007: 8).

Ähnliche oder schlimmere Berichte gibt es auch von ArbeitnehmerInnen aus Asien und dem pazifischen Raum, die nicht in der Sicherheitsbranche angestellt waren. Neben den zu zahlenden Vermittlungsgebühren und falschen Versprechungen von Arbeit in Kuwait oder Dubai, berichten Cha (2004) und Stillman (2011) von Unterernährung, dem fehlenden Zugang zu Trinkwasser, fehlender medizinischer Versorgung, mangelhafter Unterbringung, sexuellem Missbrauch sowie der Nichtauszahlung von Löhnen. Zu erwähnen ist auch, dass den Arbeitskräften de facto keine Ausstiegsmöglichkeiten offenstanden. Einmal im Krisengebiet angekommen, müssen die ArbeitnehmerInnen für ihre Rückreise selbst aufkommen, falls sie frühzeitig aus ihrem Vertrag aussteigen möchten, verwirken dadurch zudem ihren Lohn und werden nach der Ankunft in ihrem Heimatland unter Umständen von ihren GläubigerInnen konfrontiert (vgl. McCahon 2011). Cha berichtet weiter, „, their bosses were holding their passports“ (2004) und Stillman (2011) erwähnt, dass „Meridian [der Vermittler] had reportedly threatened some with more than a thousand dollars in earlytermination fines if they left".

Es ist unwahrscheinlich, dass sich entsprechende Praktiken, wie Fahrlässigkeit, betrügerische Personalbeschaffung, Inhaftierung und Ausbeutung in diesem Umfang und dieser Dauerhaftigkeit ereignen würden, wenn HauptauftragnehmerInnen ihre Arbeitskräfte unmittelbar selbst organisieren und einstellen würden. Entsprechendes attestiert auch Sam McCahon, ein US-amerikanischer Anwalt, in einer Zeugenaussage für eine Unterkommission des House Committee on Oversight and Government Reform: „prime contractors have a history of turning a blind eye to the practice and lack any motivation to get involved in mitigation efforts" (McCahon 2011: 3). Er zitiert den Vizepräsidenten eines USamerikanischen Hauptauftragnehmers mit den Worten: „We have no privity of contract with the subcontractor's employees, so it is not our problem" (McCahon 2011: 3). Diese Aussage verdeutlicht, dass der Hauptauftragnehmer juristisch gesehen keine Verantwortung trägt und keine Verpflichtungen gegenüber den Angestellten seiner SubunternehmerInnen hat. Ähnliches berichten David Isenberg und Nick Schwellenbach (2011) in einem Bericht für das Project on Government Oversight (POGO) über die Zuständigkeitsstreitigkeiten zwischen KBR, dem Hauptauftragnehmer, einem kuwaitischen Subunternehmer namens Najlaa International Catering Services und der Personalvermittlungsfirma Transtours. In diesem Fall gab es klare Indizien für Misshandlungen, Ausbeutung und Menschenhandel, wofür Najlaa Transtours verantwortlich zu machen versuchte. Obwohl KBR die Vorwürfe seit 2008 bekannt war, vergab die Firma weiterhin Aufträge an Najlaa und übernahm somit keine Verantwortung für die Zulieferkette (Isenberg/Schwellenbach 2011). 
Zusammenfassend kann man sagen, dass die die westliche Kriegsführung unterstützenden Zulieferketten einerseits vielen Menschen aus dem globalen Süden zu neuen Arbeitsplätzen verhelfen, wodurch diese ihre Familien mit Geldsendungen unterstützen können. Andererseits werden andere nachteilig in diese neue globale Wirtschaft einbezogen, was für viele hohe finanzielle, soziale und körperliche Kosten mit sich bringt. Auch wenn diese Arbeit nicht als abschließende empirische Analyse gedacht ist, zeigt sich, dass sich ein Wandel der sozialen, physischen und wirtschaftlichen Kosten und Nutzen der Kriegsführung vollzieht.

\section{Fazit}

In diesem Essay habe ich argumentiert, dass obwohl Regulierungs- und Rechenschaftsfragen in der Forschung zu PMSCs eine zentrale Rolle spielen, sich bislang wenige Arbeiten mit der Verantwortung und Haftung gegenüber den ArbeitnehmerInnen beschäftigen. Ich habe außerdem dargelegt, dass das Auslagern von Militär- und Unterstützungsdiensten und die damit verbundene Schaffung von neuen Arbeitsplätzen weder als eindeutig negativ noch positiv zu bewerten ist, da die individuellen Umstände entscheidend sind. Festzuhalten bleibt, dass die Einführung von internationalen labour supply chains sowohl die Verteilung der sozialen, physischen und wirtschaftlichen Kosten und Nutzen der Kriegsführung, als auch die Art und Weise wie Kriegspersonal organisiert wird, verändert hat.

Wie Morris Janowitz und Charles C. Moskos in Bezug auf Berufsarmeen schrieben: Die Vorstellung, dass eine Kriegslast zum Großteil von „sozial benachteiligten Teilen der Gesellschaft“ getragen wird, „entspricht nicht dem Ethos einer Demokratie“ (Janowitz/Moskos 1979: 190, eigene Übersetzung). Meiner Meinung nach sollte im Hinblick auf die derzeitige Globalisierung von privatem Kriegspersonal dieser Gedanke wieder aufgegriffen werden und die Ursachen und Folgen der Globale labour supply chains weiter untersucht werden.

\section{Literatur}

Barker, Isabella V. 2009: (Re)Producing American Soldiers in an Age of Empire, in: Politics \& Gender 5: 2, 211-235.

Barrientos, Stephanie W. 2013: Labour Chains. Analysing the Role of Labour Contractors in Global Production Networks, in: Journal of Development Studies 49: 8, 1058-1071.

Berndtsson, Joachim 2014: Realizing the ,Market-state'? Military Transformation and Security Outsourcing in Sweden, in: International Journal: Canada's Journal of Global Policy Analysis 69: 4, 542-558.

Brookings Institution 2012a: Iraq Index: Tracking Variables of Reconstruction \& Security in PostSaddam Iraq, in: https://www.brookings.edu/wpcontent/uploads/2016/07/index20120131.pdf; 31.01.2012. 
Brookings Institution 2012b: Afghanistan Index. Also including selected data on Pakistan, in: https://www.brookings.edu/wp-content/uploads/2016/07/index20120229.pdf; 29.02.2012.

Castles, Stephen 2010: Understanding Global Migration. A Social Transformation Perspective, in: Journal of Ethnic and Migration Studies 36: 10, 1565-1586.

CENTCOM - US-American Central Command 2008: Quarterly Contractor Census Reports, in: http://www.acq.osd.mil/log/ps/archvd CENTCOM reports.html; 19.11.2008.

CENTCOM - US-American Central Command 2009: Quarterly Contractor Census Reports, in: http://www.acq.osd.mil/log/ps/archvd CENTCOM reports.html; 11.2009.

CENTCOM - US-American Central Command 2010: Quarterly Contractor Census Reports, in: http://www.acq.osd.mil/log/ps/centcom reports.html; 15.12.2010.

CENTCOM - US-American Central Command 2011: Quarterly Contractor Census Reports, in: http://www.acq.osd.mil/log/ps/centcom reports.html; 17.10.2011.

CENTCOM - US-American Central Command 2012: Quarterly Contractor Census Reports, in: http://www.acq.osd.mil/log/ps/centcom reports.html; 11.2012.

CENTCOM - US-American Central Command 2017: Quarterly Contractor Census Reports, in: http://www.acq.osd.mil/log/ps/centcom reports.html; 07.2017.

Cha, Ariana E. 2004: Underclass of Workers Created in Iraq, in: Washington Post, 1.7.2004, A01.

Chief Review Services 2006: Evaluation of the Canadian Forces Contractor Augmentation Program (CANCAP), in: http://publications.gc.ca/collections/collection 2016/mdn-dnd/D58-146-2006eng.pdf; 24.6.2017.

Chisholm, Amanda 2014: Marketing the Gurkha Security Package. Colonial Histories and Neoliberal Economies of Private Security, in: Security Dialogue 45: 4, 349-372.

Chisholm, Amanda/Stachowitsch, Saskia 2016: Everyday Matters in Global Private Security Supply Chains. A Feminist Global Political Economy Perspective on Gurkhas in Private Security, in: Globalizations 13: 6, 815-829.

Coe, Neil/Hess, Martin 2013: Global Production Networks, Labour and Development, in: Geoforum 44, 4-9.

Cowen, Deborah. E. 2006: Fighting for Freedom. The End of Conscription in the United States and the Neoliberal Project of Citizenship, in: Citizenship Studies 10: 2, 167-183.

Delany, Max 2009: Why 10.000 Ugandans are Eagerly Serving in Iraq, in: The Christian Science Monitor, 6.3.2009.

Eichler, Maya 2014: Citizenship and the Contracting out of Military Work. From National Conscription to Globalized Recruitment, in: Citizenship Studies 18: 6, 600-614.

Folketing 2007: § 20-spørgsmål S 3095 Om billig arbejdskraft via Supreme Global Service Solutions, in: http://www.ft.dk/samling/20061/spoergsmaal/S3095/index.htm; 22.02.2007.

Henderson, Jeffrey/Dicken, Peter/Hess, Martin 2002: Global Production Networks and the Analysis of Economic Development, in: Review of International Political Economy 9: 3, 436-464.

Herbert, David G. 2016: Uganda's Top Export. Mercenaries, in: Bloomberg Businessweek, 11.5.2016.

Higate, P. 2012: 'Cowboys and Professionals'. The Politics of Identity Work in the Private and Military Security Company, in: Millennium - Journal of International Studies 40: 2, 321-341. 
Isenberg, David/Schwellenbach, Nick 2011: Documents Reveal Details of Alleged Labor Trafficking by KBR Subcontractor, in: POGO (Project on Government Oversight), http://www.pogo.org/ourwork/articles/2011/co-ht-20110614.html; 27.6.2017.

Janowitz, Morris/Moskos, Charles C. 1979: Five Years of the All-Volunteer Force: 1973-1979, in: Armed Forces \& Society 5: 2, 171-218.

Joachim, Jutta/Schneiker, Andrea 2015: The License to Exploit. PMSCs, Masculinities, and Third Country Nationals, in: Eichler, Maya (Hrsg.): Gender and Private Security in Global Politics, Oxford, 114128.

Kalleberg, Arne L. 2009: Precarious Work, Insecure Workers. Employment Relations in Transition, in: American Sociological Review 74: 1, 1-22.

Kapur, Devesh 2010: Diaspora, Development, and Democracy. The Domestic Impact of International Migration from India, Princeton, NJ.

Kelly, Philip F. 2013: Production Networks, Place and Development. Thinking through Global Production Networks in Cavite, Philippines, in: Geoforum 44, 82-92.

Krahmann, Elke 2010: States, Citizens and the Privatization of Security, Cambridge.

Krahmann, Elke 2013: Germany. Civilian Power Revisited, in: Leander, Anna (Hrsg.): Commercialising Security in Europe, New York, NY, 161-180.

Kruck, Andreas 2014: Theorising the Use of Private Military and Security Companies. A Synthetic Perspective, in: Journal of International Relations and Development 17: 1, 112-141.

Kruck, Andreas/Spencer, Alexander 2013: Contested Stories of Commercial Security. Self- and Media Narratives of Private Military and Security Companies, in: Critical Studies on Security 1: 3, 326346.

Lawrence, J. P. 2016: Claim: Denied. Wounded in Iraq, Ugandans fight for Compensation from America, in: The Intercept, 22.2.2016, https://theintercept.com/2016/02/22/wounded-in-iraq-ugandancontractors-fight-for-compensation-in-america/; 27.6.2017.

Leander, Anna 2013: Commercialising Security in Europe, New York, NY.

Lucena, Fernando 2013a: America's 33 Dollar Mercenaries, Pt. 1. Press TV, in https://www.youtube.com/watch?v=TA5FPI6QTIc; 14.07.2017.

Lucena, Fernando 2013b: America's 33 Dollar Mercenaries, Pt. 2. Press TV, in https://www.youtube.com/watch?v=wNiiLiKLGMQ; 14.07.2017.

Maclellan, Nic/Mares, Peter 2006: Labour Mobility in the Pacific. Creating Seasonal Work Programs in Australia (Konferenzpapier vorgestellt auf der Konferenz "Globalisation, Governance and the Pacific Islands Conference" an der Australian National University, Canberra, 25.-27.10.2005).

Mandrup, Thomas 2013: Denmark. How Not if to Outsource Military Services, in: Leander, Anna (Hrsg.): Commercialising Security in Europe, New York, NY, 39-57.

McCahon, Sam 2011: Written Testimony of Sam McCahon Before the House of Committee on Oversight and Government Reform, in: https://oversight.house.gov/wpcontent/uploads/2012/01/11-2-11 McCahon TechIP Testimony FINAL.pdf; 14.07.2017.

McCoy, Katherine E. 2010: Beyond Civil-Military Relations. Reflections on Civilian Control of a Private, Multinational Workforce, in: Armed Forces \& Society 36: 4, 671-694.

Meegdenburg, Hilde van 2015: What the Research on PMSCs Discovered and Neglected. An Appraisal of the Literature, in: Contemporary Security Policy 36: 2, 321-345. 
Miller, Christian T. 2009: Foreign Workers for U. S. Are Casualties Twice Over, in: ProPublica, 19.9.2009, https://www.propublica.org/article/foreign-workers-for-u.s.-are-casualties-twice-over-619;

27.6.2017.

Miller, Christian T. 2010: The Other Victims of Battlefield Stress. Defense Contractors' Mental Health Neglected, in: ProPublica, 26.2.2017, https://www.propublica.org/article/injured-contractorsthe-other-victims-of-battlefield-stress-224; 27.6.2017.

Mosley, Layna 2011: Labor Rights and Multinational Production, Cambridge.

Mosley, Layna/Singer David A. 2014: Migration, Labor, and the International Political Economy, in: Annual Review of Political Science 18: 1, 283-301.

Mosley, Layna/Uno, Saika 2007: Racing to the Bottom or Climbing to the Top?, in: Comparative Political Studies 40: 8, 923-948.

Olsson, Christian 2013: France. Making Both Ends Meet?, in: Leander, Anna (Hrsg.): Commercialising Security in Europe, New York, NY, 141-160.

$\emptyset$ stensen, Åse G. 2013: Norway. Keeping Up Appearances, in: Leander, Anna (Hrsg.): Commercialising Security in Europe, New York, NY, 18-38.

Páez, Ángel 2007: Perú-Iraq. Un año en el infierno por mil dólares al mes, in: IPS-Inter Press Service, 1.11.2007, $\quad$ http://www.ipsnoticias.net/2007/11/peru-iraq-un-ano-en-el-infierno-por-mildolares-al-mes/; 27.6.2017.

Perry, David 2012: Blackwater vs. bin Laden. The Private Sector's Role in American Counterterrorism, in: Comparative Strategy 31: 1, 41-55.

Petersohn, Ulrich 2010: Sovereignty and Privatizing the Military. An Institutional Explanation, in: Contemporary Security Policy 31: 3, 531-552.

Schneiker, Andrea/Joachim, Jutta 2012: Private Sicherheits- und Militärfirmen. Ein Chamäleon der internationalen Politik, in: Zeitschrift für Friedens- und Konfliktforschung 1: 1, 44-71.

Schwartz, Moshe/Swain, Joyprada 2011: Department of Defence Contractors in Afghanistan and Iraq. Background and Analysis, (herausgegeben von Congressional Research Service).

Spearin, Christopher 2003: American Hegemony Incorporated. The Importance and Implications of Military Contractors in Iraq, in: Contemporary Security Policy 24: 3, 26-47.

Spearin, Christopher 2005: Between Public Peacekeepers and Private Forces. Can There Be a Third Way?, in: International Peacekeeping 12: 2, 240-252.

Stillman, Sarah 2011: The Invisible Army, in: The New Yorker, 20.6.2011.

Stoner, Eric (2014): Outsourcing the Iraq War: Mercenary Recruiters Turn to Latin America, in: http://nacla.org/news/outsourcing-iraq-war-mercenary-recruiters-turn-latin-america; 24.6.2017.

UNHRC - United Nations Human Rights Council 2007: Report of the Working Group on the Use of Mercenaries as a Means of Violating Human Rights and Impeding the Exercise of the Right of Peoples to Self-determination, Mission to Honduras, 20.2.2007, A/HRC/4/42/Add.1, in: http://www.refworld.org/docid/461f90f22.html; 24.6.2017.

UNHRC - United Nations Human Rights Council 2008a: Report of the Working Group on the use of mercenaries as a means of violating human rights and impeding the exercise of the right of people to self-determination, 9.1.2008, A/HRC/7/7. 
UNHRC - United Nations Human Rights 2008b: Report of the Working Group on the question of the use of mercenaries as a means of violating human rights and impeding the exercise of the right of peoples to self-determination, Mission to Chile, 4.2.2008, A/HRC/7/7/Add.4.

United States Department of Labor - Office of Workers' Compensation Programs (OWCP) Defense Base Act Case Summary Reports, in: https://www.dol.gov/owcp/dlhwc/lsdbareports.htm; 14.07.2017.

\section{Die Autorin}

Dr. Hilde van Meegdenburg ist derzeit wissenschaftliche Mitarbeiterin am Geschwister-Scholl-Institut der Ludwig-Maximilians-Universität München.

E-Mail: h.vanmeegdenburg@gsi.Imu.de 